\title{
Long-term survival of exogenous embryonic stem cells in adult bone marrow
}

Cell Research (2011) 21:1148-1151. doi:10.1038/cr.2011.97; published online 14 June 2011

\section{Dear Editor,}

Embryonic stem cell (ESC) is an attractive and promising source in regenerative medicine. However, current technologies for ESC differentiation do not ensure complete or synchronized conversion of ESCs into a specific functional tissue type for therapeutic purposes. Thus, undifferentiated ESCs, though few, may be still contained in the therapeutic ESC derivatives. It is yet to be determined whether un-differentiated ESCs can survive in adult organs/tissues for a long term in vivo. Previous studies have been aimed to isolate ESC-like cells from somatic tissues or organs $[1,2]$. Embryonic remnants were thought to be "lost" during organogenesis and remain dormant in adult tissues/organs. Bone marrow (BM) has been identified as a major source containing hematopoietic stem cells (HSCs) as well as some non-hematopoietic primitive stem cells, which include mesenchymal stem cells [3], multipotent adult progenitor cells [4], marrow-isolated adult multilineage inducible cells [5], and very small embryonic-like stem cells [6]. Some of these non-hematopoietic bonemarrow-derived stem cells have been shown to have features in common with ESCs, as indicated by the expression of the ESC marker gene Oct4. The multipotent adult progenitor cells (so called "MAPCs") were once shown to differentiate into each of the three germ layer cells. However, the claim regarding the existence of ESC-like cells in adult tissues/organs such as BM remains controversial largely due to the difficulty in isolating these extremely rare cells.

In contrast to the previous studies dealing with this important yet unsettled issue, we chose to probe it by a different approach in which exogenous ESCs were directly seeded into BM cavity to see whether the BM environment was able to sustain the survival of the transplanted ESCs. In order to track the injected ESCs, we derived two different kinds of mouse ESC lines whose GFP expression was under the control of Oct4 promoter $\left(\mathrm{oGFP}^{+}\right)$or chicken $\beta$-actin promoter $\left(\mathrm{aGFP}^{+}\right)$. The fluorescent green color indicates Oct4 expression in the oGFP ${ }^{+}$cell line whereas it marks the $\mathrm{aGFP}^{+}$cell line regardless of its dif- ferentiation state. Thus, pluripotency was indicated by the presence of the oGFP ${ }^{+}$ESCs (Supplementary information, Figure S1) as opposed to the differentiation potential of the aGFP ${ }^{+}$ESCs in the recipient BM. In our experiment, mice irradiated with $700 \mathrm{cGy}$ irradiation and unirradiated mice underwent in-tibia injection of $\mathrm{oGFP}^{+} \mathrm{ESCs}$ or $\mathrm{aGFP}^{+}$ESCs (cell passages from 15 to 25 ) to minimize the peripheral loss by intravenous administration. At various time points post-transplantation, tibias, femurs, and ilia from the transplanted mice were isolated and observed using two-photon fluorescence microscopy. In addition, BM cells flushed from recipient tibias and femurs were sorted for $\mathrm{GFP}^{+}$cells and the $\mathrm{Oct}^{+}-\mathrm{GFP}^{+}$cells were re-derived as ESCs (Figure 1A).

Using two-photon microscopy, we were able to directly observe the $\mathrm{GFP}^{+}$cells and their relationship with the endosteal region in the BM. Oct $4^{+}-\mathrm{GFP}^{+}$cells stayed alive in the BM for at least 100 days after transplantation (Figure 1B). Although the time span of capturing the $\mathrm{GFP}^{+}$ cells was equally long in both irradiated and un-irradiated groups, more $\mathrm{GFP}^{+}$cells could be observed in the irradiated group and the frequency of observation of $\mathrm{GFP}^{+}$cells was higher. The irradiated microenvironment might be more conducive to the integration of grafted ESCs since irradiation could compromise or mobilize HSCs and promote blood cells to vacate from the BM niches of the recipients to make "space" for the donor cells. In addition, the roles of some unique signals from irradiated hosts in the maintenance of ESCs cannot be excluded. These may also explain why we could only recover ESC-like lines from the irradiated BM, as described below. We also found $\mathrm{GFP}^{+}$cells in the BM of the bone opposite from the injection site (Figure 1C). Two-photon microscopy and immunofluorescence results showed that most $\mathrm{GFP}^{+}$cells were randomly distributed in the injection site without physical proximity to the endosteal regions (Figure 1D). These observations suggest that the microenvironmental elements for ESCs, if present in the BM, may be different from the niche of HSCs.

Interestingly, the Oct $4^{+}$cells could be recovered at various time points post-transplantation and became cell lines 



E
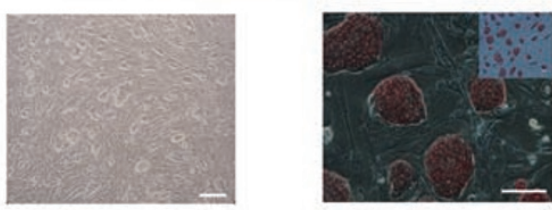
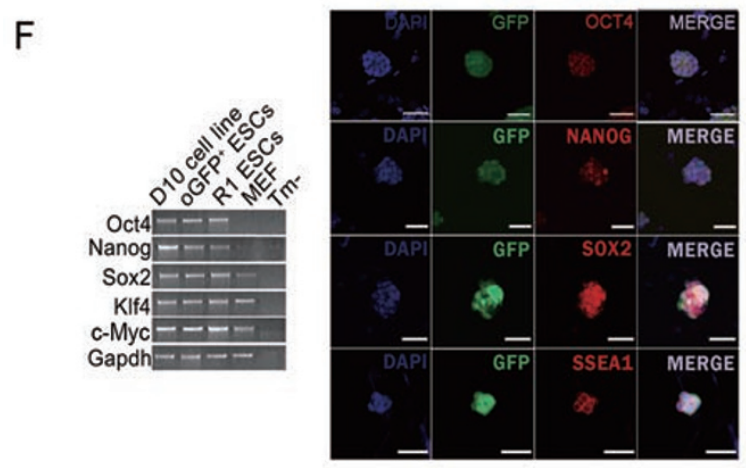

$\mathrm{H}$

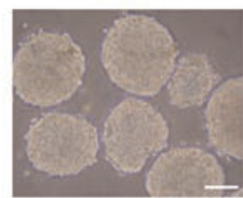

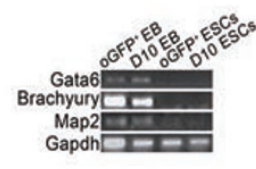

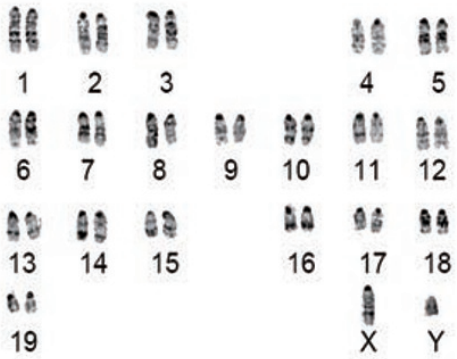

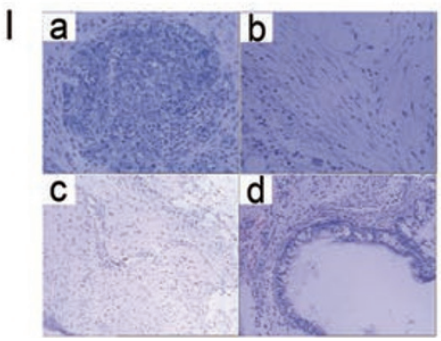

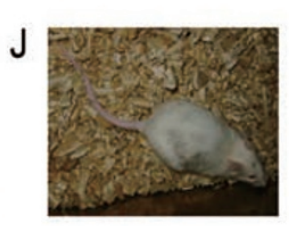

Figure 1 Tracking and recovery of exogenous GFP-marked ESCs in bone marrow (BM). (A) Experimental design. (B) Tracing of GFP ${ }^{+}$ESCs and their progeny by two-photon microscopy from different groups at different time points after transplantation. (C) $\mathrm{GFP}^{+}$cells were also found in the BM at the side of the bone opposite to the injection site in different groups. (D) Immunofluorescence of $\mathrm{GFP}^{+}$cells in BM. (E) A phase-contrast image (scale bar $=200 \mu \mathrm{m}$ ) and high levels of alkaline phosphatase activity (scale bar $=100 \mu \mathrm{m}$ ) of re-derived Oct4 ${ }^{+}$cell lines. $(\mathrm{F})$ As exemplified by the D10 cell line, re-derived Oct4 ${ }^{+}$ cell lines expressed ESC markers as assayed by fluorescent immunostaining (scale bar $=50 \mu \mathrm{m})$ and RT-PCR (R1 ESCs, MEF, and template-free PCR systems (Tm-) were used as controls). (G) Karyotype (40, XY) of re-derived D10 cell line. (H) Embryoid body formation (scale bar $=200 \mu \mathrm{m}$ ) and RT-PCR analysis of markers for three-germ layers on day-4 embryoid bodies. (I) Teratoma formation. Teratomas were harvested 4 weeks after subcutaneous injection of the D10 cell line into SCID mice. Primitive cell (a), mesodermal immature fibrous cells (b), primitive neuroectodermal cells (c), and endodermal glandular epithelium (d) were detected by hematoxylin and eosin staining. $(\mathrm{J})$ Chimeric mouse. 
again. We successfully re-derived and expanded eight cell lines from BM at multiple time points after transplantation: three cell lines on day 5 (D5), one cell line on day 10 (D10), two cell lines on day 12 (D12), one cell line on day 15 (D15), and one cell line on day 23 (D23) (Supplementary information, Table S1). Those re-established cell lines displayed high levels of alkaline phosphatase activity (Figure 1E) and expressed all markers known for ESC (Figure 1F). They had a normal karyotype of 40 XY (Figure $1 \mathrm{G})$ and were able to differentiate into cells of three germ layers (Figure 1H and 1I). In spite of all these properties similar to oGFP ${ }^{+}$ESCs, the pluripotency of these cell lines appeared to be decreased as indicated by the low chimerism (Figure 1J) and the fact that no germline transmission could be observed in the chimeric mice produced.

We compared the global gene-expression profiles of oGFP $^{+}$ESCs (passage 26), and the D5 (passage 8) and D10 (passage 7) cell lines, using DNA microarray. The microarray analyses revealed that the D5 and D10 cell lines were each clustered closely, similar to each other but a little different from the oGFP ${ }^{+}$ESCs (Supplementary information, Figure S2A). The gene expression profiles by microarray indicated little difference in most pluripotency and differentiation genes among these three groups, including: Nanog, Sox2, Klf4, Nestin, Map2, Brachyury, Bmp4, Nodal1, Pecam1, Gata4, and Gata6. However, alteration in some imprinted genes, including H19, Igf2r, Phlda2, Htr2a, Tnfrsf23, Peg10, Igf2, Sgce, Ndn, Plagl1, and Xist, was detected, suggesting that epigenetic alterations might account for the decreased pluripotency of the recovered ESCs (Supplementary information, Figure S2B). Next, we examined CpG-rich cis-elements known as differently methylated regions for important imprinted genes (Igf2-H19, Igf2r, and Xist) using bisulfite sequencing (Supplementary information, Figure S3). We found that D10 cell lines showed significant hypomethylation $(16.25 \%)$ of the differently methylated regions for the Igf2-H19 locus. In contrast, this region was slightly hypermethylated in the oGFP ${ }^{+}$ESCs and D5 cell lines. Compared to oGFP ${ }^{+}$ESCs, the D5 and D10 cell lines had the same tendency of methylation patterns in the differently methylated regions for Igf2r and Xist. Although time-dependent epigenetic alterations were shown in the re-established cell lines, it is unknown how the microenvironment of BM could alter the epigenetic status of transplanted ESCs. Thus, epigenetic instability needs to be monitored when using stem cells for technological and therapeutic purposes.

It is not clear which molecules are responsible for sustaining ESCs in BM. The bone morphogenetic proteins (BMPs) might be potential candidates [7]. BMPs act in combination with LIF to sustain self-renewal and preserve the multilineage differentiation capacity of ESCs. They also induce the expression of the inhibitor of differentiation (Id) genes, which are required for the self-renewal response produced by LIF/STAT3. This is consistent with our microarray and real-time results, which showed that Id 2 and Id 3 are expressed at higher levels in D10 cells and D5 cells than in ESCs. Alternatively, given the fact that ESCs have been shown to grow autonomously [8] and the autocrine signaling of ESC colonies produces a radial organization of self-renewal, generating a fixed-locationindependent autoregulatory niche [9], ESCs may yield their own niches to maintain their self-renewal and pluripotency in BM.

Upon injection, $\mathrm{GFP}^{+}$cells were rapidly lost in the BM, possibly caused by massive death, differentiation and migration of ESCs in the local environment. Nevertheless, a small portion of the injected ESCs, as indicated by the $\mathrm{Oct}^{+}$cells in the oGFP ${ }^{+}$ESCs-transplanted group, were able to survive and maintain their pluripotency, thereby suggesting an adaptability of ESCs in an adult environment. Recently, transplanting human ESCs into the hippocampus of athymic nude rats has been shown to improve cognitive impairment caused by irradiation. The transplanted cells can migrate to the subgranular zone and exhibit signs of neuron morphology within the neurogenic niche with no observed overt adverse cognitive sequelae or intracranial teratogenesis [10]. In contrast, our study detected teratomas in about $30 \%$ of irradiated hosts and $7.5 \%$ of unirradiated hosts. Thus, our study also offers a unique model to study how stem cell pluripotency is potentially linked to tumorigenesis.

In conclusion, our current study employed a novel approach by transplanting exogenous GFP-marked ESCs and demonstrated that the BM environment can sustain long-term survival of pluripotent stem cells, though decreased pluripotency of the transplanted ESCs may occur. This approach should be also applicable to the studies on induced pluripotent stem cells (iPSC). Therefore, our study has important implications for the understanding of stem cell development and maintenance, as well as for the safety evaluation of therapeutic ESC or iPSC derivatives, especially given the fact that rare un-differentiated ESCs can be contained in the final cellular products. Further studies are warranted regarding dissection of the microenvironment in the BM responsible for ESC survival and potential differentiation.

\section{Acknowledgments}

We are grateful to Cheng Zhan, Lanlan Gu (National Institute of Biological Sciences, China), and Jinhong Wang (Institute of Hematology, CAMS, China) for their assistance regarding two- 
photon microscopy and flow cytometric analysis. The work was supported by grants from the Ministry of Science and Technology of China (2011CB964801, 2009CB521803, 2010DFB30270, 2008AA022311, 2008AA1011005 and 2010CB944900) and research funds from the Tianjin Science and Technology Commission (07JCZDJC10600 and 09ZCZDSF03800). T.C. was a recipient of the Yangzi River Scholarship from the Ministry of Education of China (2007-JGT-08), the Outstanding Young Scholar Award from the National Natural Science Foundation of China (NSFC; 30825017), and the Scholar Award from the Leukemia and Lymphoma Society.

Yueying $\mathrm{Li}^{1,2,4}$, Yanfeng $\mathrm{Liu}^{1,4}$, Jing $\mathrm{He}^{2}$, Fengchao Wang ${ }^{2}$, Sheng Liu ${ }^{2}$, Yu Zhang ${ }^{2}$, Zhaohui $\mathrm{Kou}^{2}$, Zhenyu Ju, ${ }^{3,4}$, Guoguang Zheng ${ }^{1,4}$, Jing $\mathrm{Xu}^{1,4}$, Weiping Yuan ${ }^{1,4}$, Shaorong $\mathrm{Gao}^{2,4}$, Tao Cheng ${ }^{1,4}$

${ }^{I}$ State Key Laboratory of Experimental Hematology, Institute of Hematology and Blood Diseases Hospital, Chinese Academy of Medical Sciences and Peking Union Medical College, Nanjing Road, Tianjin 300020, China; ${ }^{2}$ National Institute of Biological Sciences, \#7 Science Park Road, Zhongguancun Life Science Park, Beijing 102206, China; ${ }^{3}$ Max-Planck-Partner Group on Stem Cell Aging, Institute of Laboratory Animal Science, Chinese Academy of Medical Sciences, Beijing, China; ${ }^{4}$ Center for Stem Cell Medicine, Chinese Academy of Medical Sciences, Beijing, China

Correspondence: Tao Cheng ${ }^{\mathrm{a}}$, Shaorong Gao ${ }^{\mathrm{b}}$

${ }^{a}$ Tel: +86-22-23909156; Fax: +86-22-23902047

E-mail: chengt@pumc.edu.cn

${ }^{\text {b}}$ Tel: +86-10-80728967; Fax: +86-10-80727535

E-mail: gaoshaorong@nibs.ac.cn

\section{References}

1 Beltrami AP, Cesselli D, Bergamin N, et al. Multipotent cells can be generated in vitro from several adult human organs (heart, liver, and bone marrow). Blood 2007; 110:3438-3446.

2 Guan K, Nayernia K, Maier LS, et al. Pluripotency of spermatogonial stem cells from adult mouse testis. Nature 2006; 440:1199-1203.

3 Friedenstein AJ, Chailakhjan RK, Lalykina KS. The development of fibroblast colonies in monolayer cultures of guinea-pig bone marrow and spleen cells. Cell Tissue Kinet 1970; 3:393-403.

4 Jiang Y, Jahagirdar BN, Reinhardt RL, et al. Pluripotency of mesenchymal stem cells derived from adult marrow. Nature 2002; 418:41-49.

5 D'Ippolito G, Diabira S, Howard GA, Menei P, Roos BA, Schiller PC. Marrow-isolated adult multilineage inducible (MIAMI) cells, a unique population of postnatal young and old human cells with extensive expansion and differentiation potential. J Cell Sci 2004; 117:2971-2981.

6 Kucia M, Reca R, Campbell FR, et al. A population of very small embryonic-like (VSEL) CXCR4(+)SSEA-1(+)Oct-4+ stem cells identified in adult bone marrow. Leukemia 2006; 20:857-869.

7 McCullough KA, Waits CA, Garimella R, Tague SE, Sipe JB, Anderson HC. Immunohistochemical localization of bone morphogenetic proteins (BMPs) 2, 4, 6, and 7 during induced heterotopic bone formation. J Orthop Res 2007; 25:465-472.

8 Ying QL, Wray J, Nichols J, et al. The ground state of embryonic stem cell self-renewal. Nature 2008; 453:519-523.

9 Davey RE, Zandstra PW. Spatial organization of embryonic stem cell responsiveness to autocrine gp130 ligands reveals an autoregulatory stem cell niche. Stem Cells 2006; 24:2538-2548.

10 Acharya MM, Christie LA, Lan ML, et al. Rescue of radiationinduced cognitive impairment through cranial transplantation of human embryonic stem cells. Proc Natl Acad Sci USA 2009; 106:19150-19155.

(Supplementary information is linked to the online version of the paper on the Cell Research website) 